\title{
HIPPOCAMPAL FREQUENCY SHIFTS IN DIFFERENT BEHAVIOURAL SITUATIONS
}

\author{
A. KAMP, F. H. LOPES DA SILVA AND W. STORM VAN LEEUWEN \\ Brain Research Group, Institute of Medical Physics, Health Research Organisation TNO, Utrecht \\ (The Netherlands)
}

(Accepted March 15th, 1971)

\section{INTRODUCTION}

In many studies of hippocampal bioelectrical correlates of behaviour special attention has been paid to two poles of hippocampal electrical activity, the theta rhythm (4-7 c/sec) and the so-called desynchronized activity2,3,8-11,13. In a few investigations emphasis has been put not on the presence or absence of theta rhythm but rather on the relevance of shifts of the dominant frequency component within the theta rhythm. In a previous investigation of Lopes da Silva and Kamp a consistent shift of hippocampal theta frequency from 5 to $6 \mathrm{c} / \mathrm{sec}$ was shown to occur at the beginning of a stereotyped behaviour pattern when a dog withdrew from a pedal in an operant behaviour situation. This finding apparently contradicted the contention of Elazar and Adey ${ }^{4}$ that such a shift was a specific correlate of approach behaviour. The contradiction might, however, be only superficial if one could envisage such a frequency shift as correlated with some other behavioural aspect rather than with approach or withdrawal per se. To investigate this possibility we tried to answer two questions: (a) whether similar shifts in hippocampal theta frequency do occur in the same animals in different behavioural situations including approach and withdrawal, and if so, (b) which is the common behavioural denominator correlated with such a shift.

To answer these questions we investigated, in approach situations, the same dogs in which the shift correlated with withdrawal from a pedal had been found. A short communication on this topic was presented by Kamp et al. ${ }^{5}$ elsewhere. We thought it might be of interest to include field situations in this study where the limitations of the laboratory paradigms could be avoided.

\section{MATERIAL AND METHODS}

Dogs with chronically implanted electrodes in the dorsal hippocampus and other brain areas were prepared as described by Lopes da Silva and Kamp ${ }^{7}$ who published details of recording. Four dogs were used. One had also been used in the study 
mentioned above. Histological confirmation of electrode placements is available for dogs.

\section{Behavioural procedures}

The field situation was devised so that a dog could be kept in his usual play environment. Dogs were allowed to play with common objects in a yard of about $2000 \mathrm{sq}$. m. It was possible to record their EEG activity in these circumstances because our radio-telemetering apparatus ${ }^{7}$ has a range with a radius of $30 \mathrm{~m}$. The field situation was set up simply by the experimenter holding in his hand an object of interest to the dog (piece of food, ball, stick); first the experimenter turned himself around with his arm well stretched out so that the dog ran in a circle around him; after several turns the experimenter threw the object to a distance of $10 \mathrm{~m}$ and the dog ran after it; at last the dog caught it and returned close to the experimenter. The behaviour was recorded on video tape simultaneously with a time code, which was also put on instrumentation tape, and eventually was filmed. A picture selected from such a film is presented in Fig. 2. The operant behavioural situation, where stereotyped withdrawal behaviour was seen, was as previously described?

\section{Analysis}

The hippocampal electrical activity was analysed on-line, or from tape, by a bank of 20 electronic bandpass filters covering the frequency range $2-32 \mathrm{c} / \mathrm{sec}$ as described by Lopes da Silva and Kamp 7 . The running average of the filters' output was either displayed in the form of a time sequence of histograms or stored on a Computer of Average Transients (CAT 400B, TMC). In the latter event 20 successive addresses of the computer were used, each corresponding to one filter. The amplitude of each filter was summed in the respective address. The output of the filters was scanned 10 times per sec. The integration time of the filter outputs in this study was typically $0.25 \mathrm{sec}$. This form of analysis can be displayed in a series of histograms with integrated filter amplitude as ordinate and frequency as abscissa. However, to present shifts in frequency within the theta band it was found preferable to transform the frequency spectra into one single value. This value will be called the coefficient of frequency shift. This value was calculated by taking from each spectrum (for instance representing the filtered activity within epochs of $2 \mathrm{sec}$ ) the amplitude of the 4 filters representing the theta band $\left(4-7 \mathrm{c} / \mathrm{sec}\right.$, i.e., $\mathrm{F}_{4}, \mathrm{~F}_{5}, \mathrm{~F}_{6}$ and $\left.\mathrm{F}_{7}\right)$, summing the lower filters' output $\left(\mathrm{F}_{4}+\mathrm{F}_{5}\right)$ and the higher ones $\left(\mathrm{F}_{6}+\mathrm{F}_{7}\right)$ and formulating the ratio between them. To avoid asymmetry around unity the logarithm of this ratio was calculated.

The coefficient of frequency shift defined in this way is given by:

$$
D=\log \frac{\left(F_{6}+F_{7}\right)}{\left(F_{4}+F_{5}\right)}
$$

This coefficient should be taken only as a convenient way of presenting shifts in frequency in the particular case under study.

Brain Research, 31 (1971) 287-294 


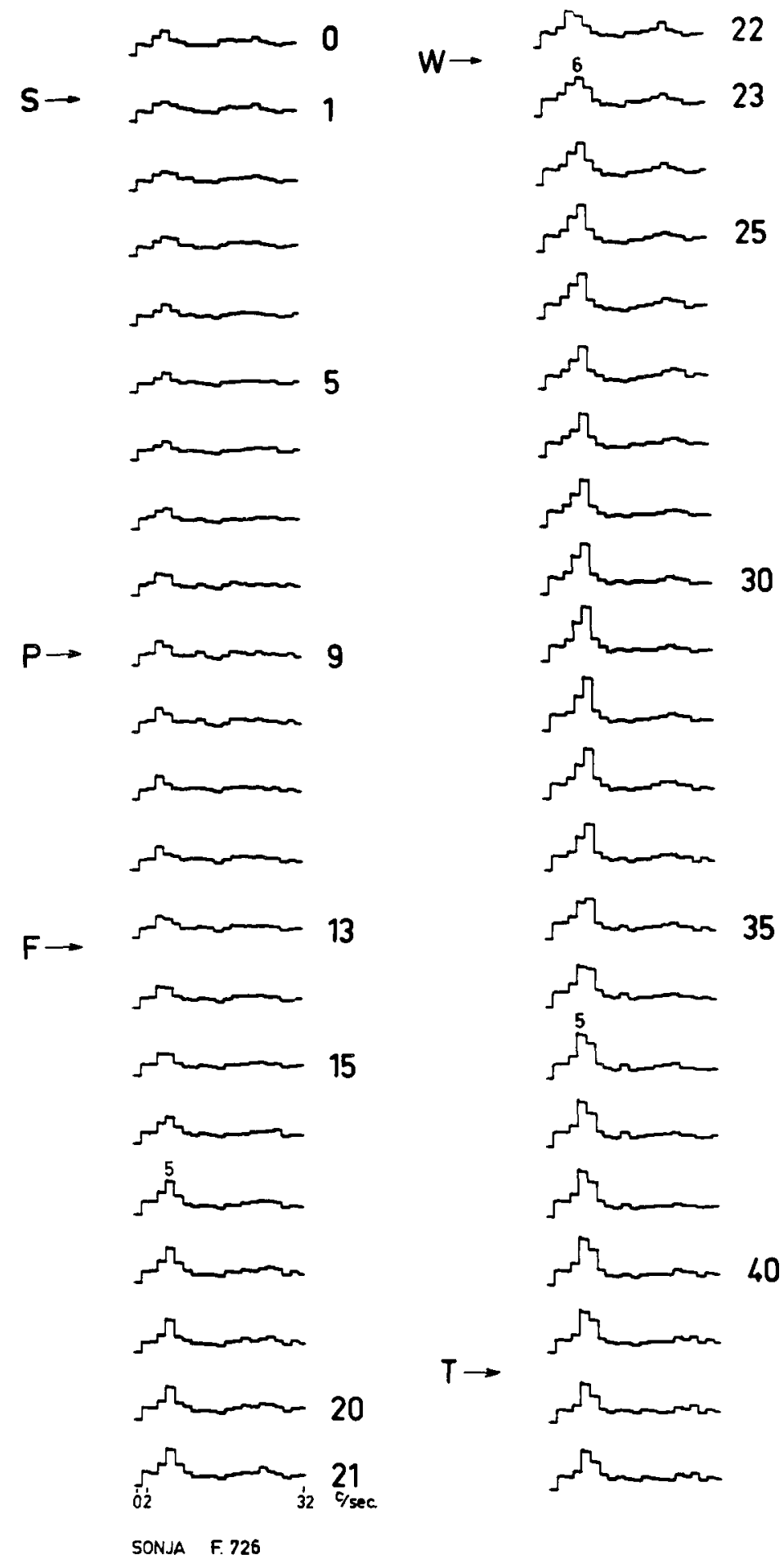

Fig. 1. Sequential frequency spectra. Time runs vertically. The interval between two histograms is 0.25 sec. Histograms start being counted with the presentation of the conditioned stimulus (S). P, dog presses the pedal; F, food is delivered; W, dog walks away from the pedal; $T$, turns back. In spectrum 21 the values of the first and last filter resonance frequencies are shown. Note low activity between $S$ and $P$. Afterwards a peak appears which is appreciable in spectrum 17 at $5 \mathrm{c} / \mathrm{sec}$, the value being indicated above the respective filter. Later the peak shifts to $6 \mathrm{c} / \mathrm{sec}$ (spectrum 23). After spectrum 36 the peak returns to $5 \mathrm{c} / \mathrm{sec}$. 


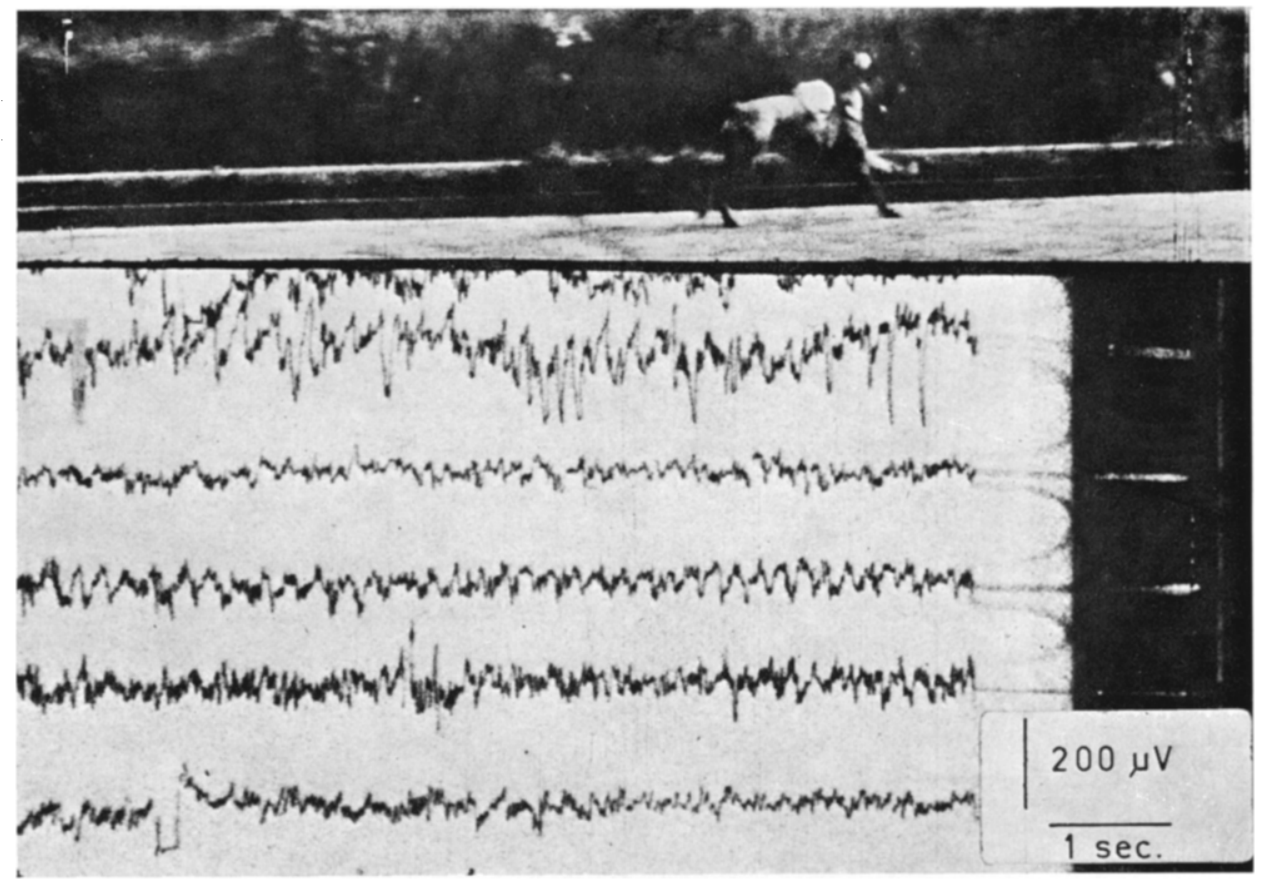

Fig. 2. Dog's behaviour illustrated with simultaneously recorded EEG activity. The picture was taken from a film montage of the dog's behaviour and of the EEG after synchronization. The phuture of the dog was taken at the same time as the moment of writing of the pens of the oscillograph. The behaviour corresponds to acts I and II (see text). The EEG was obtained by means of radio-telenetering: first trace, record of lateral eye movements; second trace, olfactory bulb; third trace, dorsal hippocampus (bipolar); fourth trace, temporal cortex; and fifth trace, amygdaloid complex (bipolar). The second and fourth recordings are against a common reference (frontal electrode).

\section{RESULTS}

The same dogs were investigated both in operant behaviour and in the field situation. In the former they typically adopted a stereotyped behavioural pattern: after the pedal pressing, in response to the conditioned stimulus, they were rewarded, then withdrew a few metres from the neighbourhood of the pedal and later returned to its proximity. The changes of hippocampal electrical activity during this behavioural sequence, which we have described precisely?, are represented in Fig. I. A typical record obtained in the field situation can be seen in Fig. 2. Electrical activities of different brain areas are shown in this figure, but our attention is here limited to those recorded from the hippocampal formation. The hippocampal activity was analysed as described in Methods, and the coefficient of frequency shift determined at successive 2 sec intervals. A plot of this coefficient for one behavioural sequence as a function of real time is shown in Fig. 3. Before analysing these values we should describe the succession of behavioural acts which invariably took place in this field situation.

These behavioural acts were as follows: first, the dog turned around; second he ran quickly after the object, third he caught it, and fourth he brought it back to the 


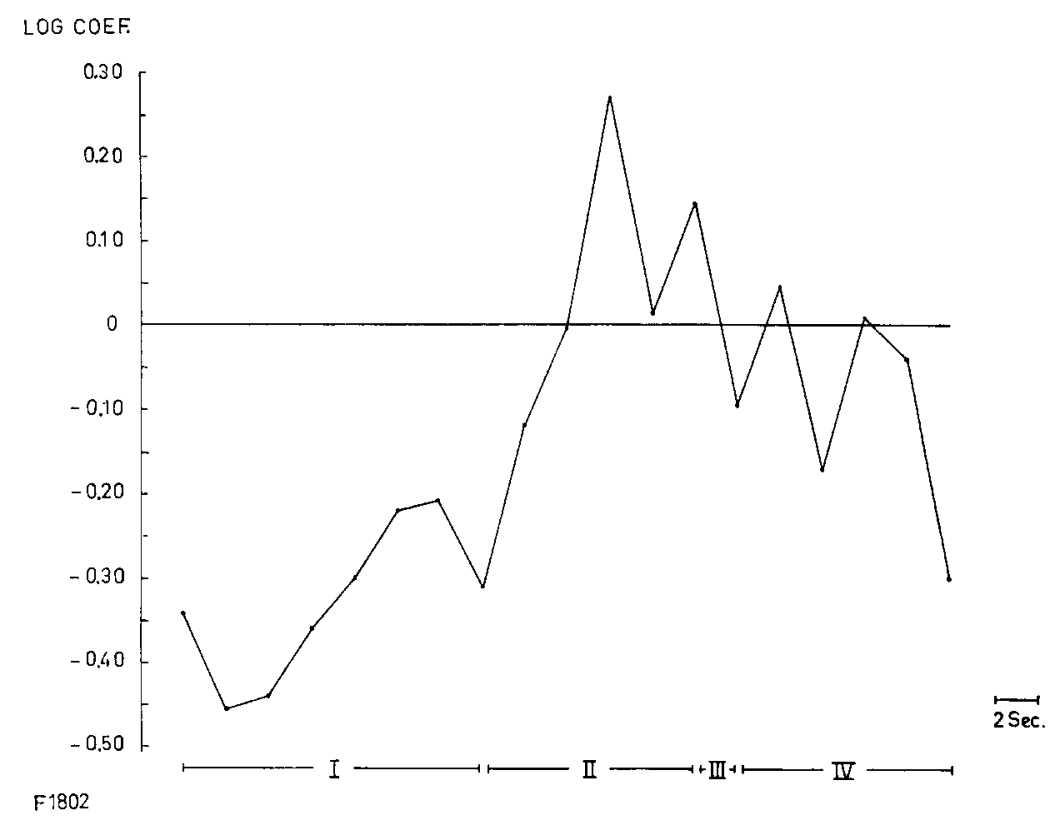

Fig. 3. Frequency shift within hippocampal theta rhythm. The roman numerals correspond to the behavioural acts described as follows: act I, the dog turns around; act II, he runs after a ball; act III, he catches it; act IV, he returns. In the ordinate the coefficient of frequency shift, defined in the text, is plotted. Note that the transition from act I to act II is accompanied by an increase of the coefficient representing a shift of the dominant theta frequency from $4-5 \mathrm{c} / \mathrm{sec}$ to $6-7 \mathrm{c} / \mathrm{sec}$. At the end of the behavioural sequence the dog returns to the experimenter and the lower frequencies $(4-5 \mathrm{c} / \mathrm{sec}) \mathrm{pre}-$ dominate.

experimenter. These successive acts are pictured in Fig. 2 and are described by roman numerals in Fig. 3. During behavioural act I (turning around) the dominant frequencies were at 4 and $5 \mathrm{c} / \mathrm{sec}$. The most striking phenomenon was the sudden shift of hippocampal theta activity to higher frequencies the moment the dog started running after the object (act I $\rightarrow$ act II). Acts III and IV were accompanied by a less stable hippocampal activity, but at the end of the behavioural sequence the low frequencies became dominant once more. It should be stressed that all along this behavioural sequence the hippocampal activity was typically characterized by theta rhythm and not by desynchronized activity. This pattern of changes of hippocampal activity and its correlation with behavioural acts were seen consistently in 4 dogs. The only differences from dog to dog were found in the precise timing of the different acts. The striking aspect, however, was that the moment the dog changed from behavioural act I to II there was a rapid shift of theta rhythm towards higher frequencies (6 and 7 $\mathrm{c} / \mathrm{sec}$ ). Whether the object thrown was a ball, a piece of food or a stick made no difference in the hippocampal activity.

\section{DISCUSSION}

The present experiments demonstrate that the theta rhythm frequency of the 
dorsal hippocampus in dogs does shift to higher values in a field situation when a dog switches from a 'turning around' type of activity to an act performed 10 catch an object thrown some distance away. This shift is similar to that found when dogs withdraw from a pedal in an operant behaviour situation. The only differences are that in the field situation the shift lasts longer and is more pronounced. Therefore. we conclude that such shifts in hippocampal theta rhythm frequency can occur in different behavioural situations. This, then, is the answer to the first question in the Introduction. The second question leads us to investigate which common behavioural denominator can be correlated with such frequency shifts. Approach and withdrawat, defined objectively in a topological sense, do not appear to be the relevant denominators because, whereas in the field situation here investigated, as well as in that of Elazar and Adey ${ }^{4}$, approach is the relevant correlate of the $5 \rightarrow 6 \mathrm{c} / \mathrm{sec}$ shift, in the case of operant behavioural situation this was not so. Movement as such is also not the common denominator because from an examination of the field situation, it is obvious that the two modes of hippocampal theta rhythm -..- low and high frequency modes -.... are both compatible with overt movement since the first accompanies 'turning around' and the second 'running after an object'.

The association of hippocampal theta activity with voluntary movement has, of course, been described several times in different species, namely in the dog by Storm van Leeuwen et al. ${ }^{11}$ and by Dalton and $\mathrm{Black}^{3}$, in the rat by Pickenhain and Klingberg $^{9}$ and by Vanderwolf ${ }^{13}$, in the guinea-pig by Sainsbury ${ }^{10}$ and in the cat by Parmeggiani $^{8}$, although Bennett ${ }^{1}$ has reported negative results in the cat. However. we have here to discuss the behavioural correlates of the frequency shift within the theta band rather than the occurrence of theta activity as such.

In the third place we should perhaps examine the proposition that shifts to higher theta frequencies may be correlated with complex behavioural functions, memory consolidation, decision making ${ }^{4}$ and orienting responses ${ }^{2}$. However, we would rather refrain from entering such speculations because such behavioural functions lack explicit definitions. We find it more attractive to see as the common"denominator of the hippocampal shifts the sudden transition, or switching, from one ${ }_{k}^{r}$ behavioural act to another occurring within a behavioural mode in which the dog is walking or running. In the field situation the dog switches from a 'turning around' to a 'running after' act. In the operant condition the dog switches from 'pressing' to 'walking away'. This type of switch in behavioural pattern is probably associated with a stepwise change of the input to the hippocampus. Then the shift in hippocampal theta frequency could be a sort of response of the septum-hippocampal neural system, to a stepwise change of its input. The latter might be mainly coming from the mesencephalic reticular formation. Therefore the step input would consist of a sudden change of reticular formation activity and the response, somewhat non-linear, would be a shift in frequency of hippocampal activity. This proposal is consistent with the observation of Stumpf ${ }^{12}$ that the frequency of the hippocampal theta rhythm can be a sensitive index of the degree of reticular excitation.

Vanderwolf ${ }^{13}$ also remarked that the theta frequency of the hippocampus increased before the occurrence of some movements, and similar results were obtained 
by Pickenhain and Klingberg 9 . Moreover $\mathrm{Klemm}^{6}$ has demonstrated that phasic multiple unit increases in midbrain reticular formation activity precede the onset of movements, or increases of muscle tone, and the occurrence of theta rhythm in rabbits. Vanderwolf also envisages that theta frequency shifts may be due to increased activity in the brain stem. In conclusion our observation leads to an interpretation of hippocampal shifts of theta rhythm within the same general frame of concepts as the above-mentioned authors $6,9,13$ have discussed in relation to the occurrence of theta rhythms.

To establish the behavioural denominator of the hippocampal shift in the terms stated above does not mean, however, that the significance of such shifts in terms of hippocampal function has been defined. This is at present open to speculation.

\section{SUMMARY}

Electrical activity of the dog's hippocampus was recorded in (a) an operant behaviour situation, and (b) a field situation by a radio-telemetering system. The dominant frequency of the theta rhythm shifted consistently from 4-5 $\mathrm{c} / \mathrm{sec}$ to $6-7$ $\mathrm{c} / \mathrm{sec}$ when a dog (a) withdrew from a pedal after being rewarded or (b) switched from a 'turning around' type of activity to an act performed to catch an object thrown some distance away.

The frequency shift was quantified by integrated frequency analysis. Such a frequency shift is interpreted in behavioural terms by considering its appearance in different behavioural situations. The correlation between such shifts and switching from one behavioural act to another, within a behavioural mode characterized by movement, is stressed.

\section{REFERENCES}

1 BenNeTt, T. L., Evidence against the theory that hippocampal theta is a correlate of voluntary movement, Commun. Behav. Biol., A4 (1969) 165-169.

2 BenNETT, T. L., Hippocampal EEG correlates of behavior, Electroenceph. clin. Neurophysiol., 28 (1970) 17-23.

3 Dalton, A., AND Black, A. H., Hippocampal electrical activity during the operant conditioning of movement and refraining from movement, Commun. Behav. Biol., A2 (1968) 267-273.

4 Elazar, Z., AND ADEY, W. R., Spectral analysis of low frequency components in the electrical activity of the hippocampus during learning, Electroenceph. clin. Neurophysiol., 23 (1967) 225-240.

5 Kamp, A., Lopes da Silva, F. H., and Storm van Leeuwen, W., Common aspects of hippocampus electrical activities during different behavioural tasks, Brain Research, 24 (1970) 547-548.

6 Klemm, W. R., Correlation of hippocampal theta rhythm, muscle activity, and brain stem reticular formation activity, Commun. Behav. Biol., A5 (1970) 147-151.

7 Lopes Da Silva, F. H., AND Kamp, A., Hippocampal theta frequency shifts and operant behaviour, Electroenceph. clin. Neurophysiol., 26 (1969) 133-143.

8 Parmeggiani, P. L., On the functional significance of the hippocampal theta rhythm. In W. R. Adey ANd T. Tokizane (Eds.), Structure and Function of the Limbic System, Progress in Brain Research, Vol. 27, Elsevier, Amsterdam, 1967, pp. 413-441.

9 Pickenhain, L., and Klingberg, F., Hippocampal slow wave activity as a correlate of basic behavioural mechanisms in the rat. In W. R. AdEY AND T. TOKIZANE (Eds.), Structure and Function of the Limbic System, Vol. 27, Elsevier, Amsterdam, 1967, pp. 218-227. 
10 Sainsbury, R. S., Hippocampal activity during natural behavior in the guinea pig, Phyiol. Behar. 5 (1970) $317-324$.

11 Storm van Leeuwen, W., Kamp, A., Kok, M. L., De Quartel, F., Lopes da Silva, F. H., 1.1 Tielen, A. M., Relations entre les activités électriques cérébrales du chien, son comportement et sa direction d'attention, Actualités neurophysiol., 7 (1967) 167-186.

12 Stumpf, $\mathrm{CH}$., Drug action on the electrical activity of the hippocampus, Int. Res. Veurobiol, 8 (1965) 77-138.

13 VANDERwolf, C. H., Hippocampal electrical activity and voluntary movement in the rat, Electroenceph. clin. Neurophysiol., 26 (1969) 407-418. 